\title{
Nuevos escenarios normalizados para la integración de la responsabilidad social y la prevención de riesgos laborales en el sistema de gestión empresarial
}

\author{
Francisco Brocal ${ }^{a}$, Irene Bajo y Pedro Varóc
}

DOI: $10.12961 /$ aprl.2019.22.02.4

\section{RESUMEN}

En los campos específicos de la gestión de la prevención de riesgos laborales (PRL) y la responsabilidad social (RS) han surgido en los últimos meses importantes novedades con la aprobación de la norma ISO 45001:2018 sobre sistemas de gestión de la seguridad y salud laboral, así como con la publicación en materia de RS del ISO International Workshop Agreement-IWA 26:2017, sobre el uso de la norma ISO 26000:2010 para aquellas organizaciones que han implementado una o más normas ISO sobre sistemas de gestión. Todo ello define nuevos escenarios de normalización, siendo especialmente novedoso el escenario vinculado a la norma ISO 45001:2018 e IWA 26:2010, el cual presenta nuevas oportunidades a través de la integración de los marcos de gestión en materia de PRL y RS.

PALABRAS CLAVE: Gestión, riesgo laboral, responsabilidad social, ISO 45001, ISO 26000, IWA 26.

\section{NEW STANDARDIZED SCENARIOS FOR THE INTEGRATION OF SOCIAL RESPONSIBILITY AND THE OCCUPATIONAL RISK PREVENTION INTO A BUSINESS MANAGEMENT SYSTEM}

\begin{abstract}
In the specific fields of occupational safety and health (OSH) and social responsibility (SR) management, important developments have emerged in recent months following the approval of ISO 45001:2018 on OSH management and the publication on SR of the ISO International Workshop Agreement-IWA 26: 2017 on the use of the ISO 26000:2010 standard for those organizations that have implemented one or more ISO standards on management systems. This creates new standardization scenarios, one of the most novel being the scenario linked to ISO 45001:2018 and IWA 26:2010, which presents new opportunities through the integration of management frameworks in OSH and SR.
\end{abstract}

KEYWORDS: Management, occupational risk, social responsibility, ISO 45001, ISO 26000, IWA 26.

\section{INTRODUCCIÓN}

Uno de los objetivos fundamentales de la gestión de la prevención de riesgo laborales (PRL) es sin lugar a dudas su integración en el sistema general de gestión de las organizaciones. Al respecto, la Ley 31/1995 de PRL (LPRL) ${ }^{1}$ establece que la política en materia de PRL deberá promover la integración eficaz de la prevención en el sistema de gestión de la empresa, comprendiendo según el RD 39/1997 por el que se aprueba el reglamento de los Servicios de Prevención (RSP) ${ }^{2}$, tanto al conjunto de las actividades como a todos sus niveles jerárquicos.

a. Departamento de Física, Ingeniería de Sistemas y Teoría de la Señal,

Universidad de Alicante, Alicante, España.

b. Departamento de Derecho del Trabajo y de la Seguridad Social,

Universidad de Alicante, Alicante, España.

c. Departamento de Ingeniería Química, Universidad de Alicante,

Alicante, España.
Dicho proceso de integración requiere del desarrollo de un sistema de gestión de la PRL (SGPRL) de carácter obligatorio con objeto de dar respuesta al marco jurídico configurado por la LPRL, el RSP y demás normativa de desarrollo. Consecuentemente, este SGPRL deberá integrarse y en su caso complementarse con otros sistemas de gestión de la empresa.

\section{SISTEMAS DE GESTION NORMALIZADOS}

Las organizaciones pueden adoptar de forma voluntaria un sistema de gestión "normalizado", entendiéndose como tal y con carác-

\footnotetext{
Correspondecia:

Francisco Brocal

Departamento de Física, Ingeniería de Sistemas y Teoría de la Señal,

Universidad de Alicante, Alicante, España.

francisco.brocal@ua.es
} 
y la prevención de riesgos laborales en el sistema de gestión empresarial

ter general, aquel sistema que cumple los requisitos establecidos en una norma ${ }^{3}$. Entre los sistemas de gestión normalizados más conocidos a nivel internacional, se encuentran los vinculados a la calidad, medio ambiente y PRL, a través de las normas ISO 9001:20154, ISO 14001:2015 y OSHAS 18001:2007 ${ }^{6}$, respectivamente.

En materia de responsabilidad social (RS) se cuenta con la norma ISO 26000:20107, la cual no es un sistema de gestión propiamente dicho, sino una guía que permite integrar la RS dentro de una organización. En los campos específicos de la gestión de la PRL y la RS han surgido en los últimos meses importantes novedades con la aprobación de la norma ISO 45001:2018 sobre sistemas de gestión de la seguridad y salud laboral, así como con la publicación en materia de RS del ISO International Workshop Agreement-IWA 26:2017 , sobre el uso de la norma ISO 26000:2010 para aquellas organizaciones que han implementado una o más normas ISO sobre sistemas de gestión. La principal diferencia entre una norma ISO y un documento IWA, radica en que el segundo recoge un acuerdo internacional desarrollado fuera del sistema normal de los comités de $\mathrm{ISO}^{10}$. En relación al término "norma" conviene subrayar que debe entenderse en el sentido del artículo 5.3 del RD 39/1997.

\section{NUEVOS ESCENARIOS NORMALIZADOS}

La norma ISO 45001:2018 e IWA 26:2010 configuran un nuevo escenario normalizado para la PRL y la RS, el cual genera nuevas oportunidades para la mejora de la gestión empresarial de ambas materias. La publicación de ISO 45001:2018 supone la anulación de OHSAS 18001:2007, por lo que las empresas certificadas según este estándar disponen de tres años para hacer la migración (Contreras, 2018) ${ }^{11}$. Existen muchas diferencias entre la ISO 45001:2018 y OHSAS 18001:2001, pero la principal novedad es que la ISO 45001:2018 se centra en la interacción entre una organización y su entorno de negocio, mientras que el estándar OHSAS 18001 pone el énfasis en la gestión de los riesgos en materia PRL y otros aspectos internos (Glaesel y Corrie, 2018) ${ }^{12}$. La implementación de ISO 45001:2018 aporta numerosos beneficios, entre los que se encuentran conseguir una mayor optimización en la gestión de la PRL así como mejorar la imagen de la empresa al demostrar a sus partes interesadas, su responsabilidad y compromiso con la seguridad y salud (Campos et al., 2018) ${ }^{13}$.

Los modelos de PRL y RS se encuentran íntimamente relacionados, de forma que tal y como consideran Bestratén y Pujol $(2003)^{14}$, con carácter general la PRL es uno de los capítulos fundamentales de la RS. De hecho, la norma ISO 26000:2010 recoge como uno de los beneficios de la RS para una organización la mejora de la salud y la seguridad de sus trabajadores y trabajadoras. Al respecto, la norma indica que las organizaciones deberían revisar sus decisiones y actividades para eliminar sesgos de género y promover igualdad de género, incluyendo entre otros, los posibles impactos diferenciadores en hombres y mujeres en lo que se refiere al lugar de trabajo y la salud y la seguridad de la comunidad.

Desde una perspectiva más amplia y general, la norma ISO 26000:2010 considera que una organización para definir su RS,

Arch Prev Riesgos Labor 2019; 22 (2): 81-83 identificar asuntos pertinentes y establecer sus prioridades, debería abordar las denominadas materias fundamentales, siendo: gobernanza de la organización; derechos humanos; prácticas laborales; medio ambiente; prácticas justas de operación; asuntos de consumidores; y participación activa de la comunidad.

Cada una de estas materias fundamentales está organizada en torno a un conjunto de los denominados asuntos. De esta forma, las materias vinculadas a la gobernanza de la organización, los derechos humanos (asunto 8), prácticas laborales (asuntos 2, 3, 4 y 5), medio ambiente (asunto 1), consumidores (asuntos 2 y 7 ) y participación activa y desarrollo de la comunidad (asunto 6), abordan directamente aspectos propios de la PRL. En cuanto a la materia de las prácticas laborales, puede considerarse que en su conjunto aborda aspectos propios de la PRL, aunque resulta particularmente de interés su asunto 4 sobre salud y seguridad ocupacional. Así, se observa que la PRL adopta un carácter transversal en la norma ISO 26000:2010, aunque es a través de la materia de las prácticas laborales donde se desarrolla de forma más específica.

Por otra parte, tomando como referencia las materias fundamentales indicadas, De Nicolais ${ }^{15}$ analizó 6 modelos de gestión de la PRL y 21 modelos de gestión de la RS, entre los que se encuentran los modelos derivados de la LPRL, ISO 45001:2018 e ISO 26000:2010. Entre los 21 modelos de gestión de la RS, dicho autor identificó, analizó y clasificó 100 factores vinculados con la PRL. Entre los criterios utilizados en la clasificación, De Nicolais ${ }^{15}$ consideró las materias fundamentales abordadas según ISO 26000:2010, obteniendo entre otros resultados que la materia correspondiente a las prácticas laborales es la que mayor influencia ejerce sobre la PRL, lo cual es coherente con las características de esta materia fundamental.

Entre estos 100 factores clasificados por materias según ISO 26000:2010, puede resultar especialmente de interés estudiar a través de futuros trabajos, aquellos que impulsan la RS a través de la integración de sistemas de gestión de la PRL, considerando para ello el marco jurídico de carácter obligatorio establecido por la LPRL y normativa de desarrollo, así como los nuevos marcos voluntarios definidos por la ISO 45001:2018 e ISO 26000:2010.

En todo caso, diseccionar la norma ISO 26000:2010 con objeto de identificar materias y asuntos vinculados a la PRL, puede ser un ejercicio complejo que difícilmente lleve a resultados exhaustivos, debido precisamente al componte transversal de la PRL apuntado con anterioridad, y por supuesto también debido al amplio espectro de la RS. Por ejemplo, la materia fundamental sobre el medio ambiente, en principio está vinculado a la gestión ambiental y no a la gestión de la PRL. Sin embargo, aspectos específicos de la normativa de desarrollo de la LPRL establecen vínculos entre ambos marcos, como por ejemplo ocurre con el artículo 5.5.d del RD 665/1997 sobre sobre la protección de los trabajadores contra los riesgos relacionados con la exposición a agentes cancerígenos durante el trabajo ${ }^{16}$.

Realizar el camino inverso anterior, es decir, diseccionar la norma ISO 45001:2018 con el objetivo de buscar vínculos con ISO 26000:2010, no es posible llevarlo a cabo de una forma di- 
recta, ya que la primera norma advierte que no incluye requisitos específicos tales como la gestión de la RS, gestión de la calidad o gestión ambiental, aunque sus elementos pueden alinearse o integrarse con otro sistemas de gestión. En otras palabras, tal y como se ha indicado anteriormente, las normas ISO 45001:2018 e ISO 26000:2010 son susceptibles de integración, aunque para ello debe trazarse un recorrido indirecto, para lo cual puede utilizarse el documento IWA 26:2017, ya que el mismo define los vínculos y relaciones entre ISO 26000:2010 y los sistemas de gestión desarrollados bajo una estructura de alto nivel, como es el caso de ISO 45001:2018, ISO 9001:2015 o ISO 14001:2015 (a grandes rasgos, una estructura de alto nivel es una estructura común que facilita la integración entre sistemas de gestión normalizados).

\section{CONCLUSIONES}

En la actualidad se abren nuevos escenarios de normalización vinculados a la norma ISO 26000:2010 y el IWA 26:2010. Entre ellos, resulta especialmente novedoso el escenario vinculado a la norma ISO 45001:2018 e IWA 26:2010, el cual presenta nuevas oportunidades a través de la integración de los marcos de gestión en materia de PRL y RS. Uno de los primeros pasos que puede facilitar el diseño de un nuevo modelo de integración, es la identificación de factores impulsores de la RS a través de la PRL, considerando para ello como punto de partida los 100 factores estudiados por De Nicolais ${ }^{15}$. Para tal fin, de las siete materias fundamentales descritas por ISO 26000:2010, las materias vinculadas a la gobernanza de la organización, los derechos humanos, prácticas laborales, medio ambiente, consumidores y participación activa y desarrollo de la comunidad, abordan directamente aspectos propios de la PRL, cuyos vínculos reglamentarios deben estudiarse en el marco de la LPRL, así como sus vínculos voluntarios pueden analizarse en el marco de la ISO 45001:2018, para lo cual IWA 26:2010 resulta una herramienta fundamental, ya que define el hilo conductor -bidireccional- entre ISO 26000:2010 e ISO 45001:2018.

\section{AGRADECIMIENTOS}

Este trabajo ha sido financiado por la Conselleria de Transparencia, Responsabilidad Social, Participación y Cooperación de la Generalitat Valenciana, y el Vicerrectorado de Investigación y Transferencia del Conocimiento de la Universidad de Alicante. Título de la investigación: "Análisis de factores impulsores de la responsabilidad social (ISO 26000) a través de la integración de sistemas de gestión de la prevención de riesgos laborales: marco legal y normalizado según ISO 45001" con referencia TSPU. $\mathrm{NI} / 2018 / 03 / 15$.

\section{BIBLIOGRAFÍA}

1. Ley de Prevención de Riesgos Laborales. L. No 31/1995 (8 Nov 1995).

2. Real Decreto 39/1997, por el que se aprueba el Reglamento de los Servicios de Prevención (31 Ene 1997).

3. Instituto Nacional de Seguridad e Higiene en el Trabajo (INSHT). Guía Técnica para la integración de la prevención de riesgos laborales en el sistema general de gestión de la empresa (Real Decreto 604/2006, de 19 de mayo). Madrid: Instituto Nacional de Seguridad e Higiene en el Trabajo (INSHT); 2015.

4. International Organization for Standardization (ISO). ISO 9001:2015. Quality management systems-Requirements. Geneva: International Organization for Standardization (ISO); 2015.

5. International Organization for Standardization (ISO). ISO 14001. Environmental management systems-Requirements with guidance for use. Geneva: International Organization for Standardization (ISO); 2015.

6. The British Standards Institution (BS). OHSAS 18001:2007 - Occupational Health and Safety Management (OHS). London: The British Standards Institution; 2007.

7. International Organization for Standardization (ISO). ISO 26000 Social responsibility. Geneva: International Organization for Standardization; 2010.

8. International Organization for Standardization (ISO). ISO 45001:2018 Occupational health and safety. Geneva: International Organization for Standardization; 2018.

9. International Organization for Standardization (ISO). IWA 26: using ISO 26000 with management systems. Geneva: International Organization for Standardization; 2017.

10. International Organization for Standardization (ISO). 2018. [citado 16 Oct 2018]. Disponible en: https://www.iso.org/deliverables-all.html.

11. Contreras S. Cómo implantar ISO 45001. Revista de la Asociación Española de la Normalización, UNE. [edición electrónica]. 2018 [citado 22 oct. 2018]; 319 [aprox. 10 p]. Disponible en: https://revista.aenor.com/335/como-implantar-iso-45001.html?utm_medium=EMAIL\&utm_source=ES_AENOR\&utm_ campaign=A04042018070820.

12. Glaesel K, Corrie C. Todo lo que hay que saber sobre la ISO 45001 . ISO Focus. [edición electrónica]. 2018 [citado 22 oct. 2018]; [aprox. 3 p]. Disponible en: https://revista.une.org/2/todo-lo-que-hay-que-saber-sobre-la-iso-45001.html.

13. Campos F, López MA, Martínez M, Ossorio JR, Pérez JF, Rodríguez MD et al. Guía para la implementación de la norma ISO 45001 "Sistemas de gestión de la seguridad y salud en el trabajo”. Madrid: FREMAP, Mutua Colaboradora con la Seguridad Social No 61; 2018.

14. Bestratén M, Pujol L. NTP 643: Responsabilidad social de las empresas (I): conceptos generales. Instituto Nacional de Seguridad e Higiene en el Trabajo. [edición electrónica]. 2003 [citado 22 oct. 2018]; [9 p]. Disponible en: http:// www.insht.es/InshtWeb/Contenidos/Documentacion/FichasTecnicas/NTP/ Ficheros/601a700/ntp_643.pdf.

15. De Nicolais, A. Análisis de los factores de la responsabilidad social vinculados con la integración y promoción de la prevención de riesgos laborales en la empresa. Alicante: Universidad de Alicante; 2018.

16. Real Decreto 665/1997 sobre la protección de los trabajadores contra los riesgos relacionados con la exposición a agentes cancerígenos durante el trabajo (24 May 1997). 